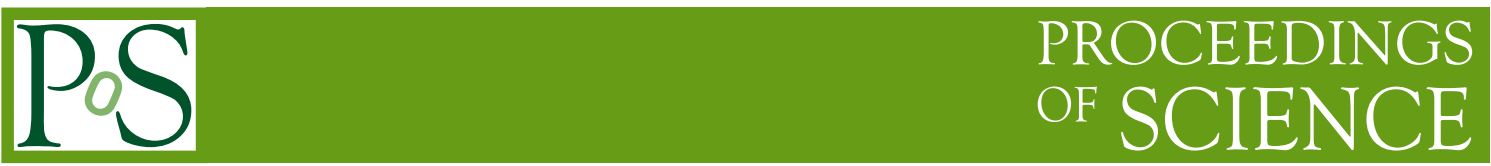

\title{
Hadronic resonance production measured by ALICE at the LHC
}

\section{Paraskevi Ganoti ${ }^{a}$, on behalf of the ALICE Collaboration}

${ }^{a}$ Department of Physics, National and Kapodistrian University of Athens, Panepistimioupolis Zografou, 15784 Athens, Greece

E-mail: pganoti@phys.uoa.gr

Hadronic resonances with different lifetimes are very useful to probe the hadronic phase of heavyion collisions. Due to their relatively short lifetimes compared to the duration of the hadronic phase, resonances are good candidates to investigate the interplay between particle re-scattering and regeneration in the hadronic phase. In addition, having different masses, quantum numbers and quark content, hadronic resonances carry a wealth of information on different aspects of ion-ion collisions, including the processes that determine the shapes of particle momentum spectra, strangeness production, and the possible onset of collective effects in small systems. We here present the latest results on resonance production in $\mathrm{pp}, \mathrm{p}-\mathrm{Pb}, \mathrm{Pb}-\mathrm{Pb}$ and $\mathrm{Xe}-\mathrm{Xe}$ collisions at different energies. Results include system-size and collision-energy evolution of transverse momentum spectra, integrated yields, mean transverse momenta and particle ratios. These results will be given through comparisons to measurements from lower energy and theoretical models.

40th International Conference on High Energy physics - ICHEP2020

July 28 - August 6, 2020

Prague, Czech Republic (virtual meeting) 


\section{Introduction}

High-energy heavy-ion (A-A) collisions provide an excellent tool to study nuclear matter under extreme conditions of temperature and energy density and the phase transition to a deconfined state of quarks and gluons, the quark-gluon plasma (QGP) predicted by lattice QCD calculations [1,2]. Similar measurements to those in A-A collisions are performed in lighter systems such as $\mathrm{p}-\mathrm{A}$ and $\mathrm{pp}$, serving as the reference for the phenomena observed. In addition, the study of these systems has revealed an interesting and unexpected behaviour of various properties, suggesting that $\mathrm{p}-\mathrm{A}$ collisions are not simply an incoherent superposition of the pp ones.

Hadronic resonances provide a unique opportunity to shed light on the particle production mechanisms and the dynamic evolution of the system formed in heavy-ion collisions. Depending on the resonance lifetime, their yields may be influenced by interactions during the late hadronic phase in $\mathrm{A}-\mathrm{A}$ and $\mathrm{p}-\mathrm{A}$ collisions. Two competing effects are expected: the re-scattering of the resonance decay products in the medium resulting in a lower detected yield and the pseudo-elastic collisions that can regenerate them. The strengths of the re-scattering and regeneration effects depend on the scattering cross sections of the decay products, the particle density of the produced medium, the lifetimes of the resonances and the lifetime of the hadronic phase. The systematic study of resonances with different decay times in different collision systems and especially their yield ratios to long-lived particles can help us to understand the interactions with the medium and to determine the lifetime of the hadronic phase ([3]).

The ALICE experiment has measured baryonic and mesonic resonances with different lifetimes and strange quark content (Table 1). Selected results from pp, $\mathrm{p}-\mathrm{A}$ and $\mathrm{A}-\mathrm{A}$ collisions are presented [3-9]. For the first time, we measure the $\Sigma(1385)^{ \pm}$resonances reconstructed in $\mathrm{Pb}-\mathrm{Pb}$ collisions in three centrality classes.

Table 1: Hadronic resonances and their lifetimes.

\begin{tabular}{|c|c||c|c||c|c||c|c||}
\hline Resonance & $\tau(\mathrm{fm} / c)$ & Resonance & $\tau(\mathrm{fm} / c)$ & Resonance & $\tau(\mathrm{fm} / c)$ & Resonance & $\tau(\mathrm{fm} / c)$ \\
\hline \hline$\rho(770)$ & 1.3 & $\mathrm{~K}^{*}(892)^{ \pm}$ & 3.6 & $\mathrm{~K}^{*}(892)^{0}$ & 4.2 & $f_{0}(990)$ & large unc. \\
\hline$\Sigma(1385)^{ \pm}$ & $5.0-5.5$ & $\Xi(1820)^{ \pm}$ & 8.1 & $\Lambda(1520)$ & 12.6 & $\Xi(1530)^{0}$ & 21.7 \\
\hline$\Lambda(1520)$ & 12.6 & $\Xi(1530)^{0}$ & 21.7 & $\phi(1020)$ & 46.4 & & \\
\hline
\end{tabular}

\section{Resonances reconstruction}

The ALICE detector [10] is designed to identify particles over a wide momentum range in the high-multiplicity environment achieved in central A-A collisions at LHC energies by using different particle identification (PID) techniques. Resonances are reconstructed via invariant mass analysis, by requiring the decay daughters to be identified by combining the relevant PID methods in momentum ranges. In Fig. 1, an example is presented for $\Sigma(1385)^{ \pm}$reconstruction in their hadronic decay channel $(\Lambda, \pi)$. In this figure, the mixed-event subtracted distribution is shown, fitted with a residual background and a Breit-Wigner function. 

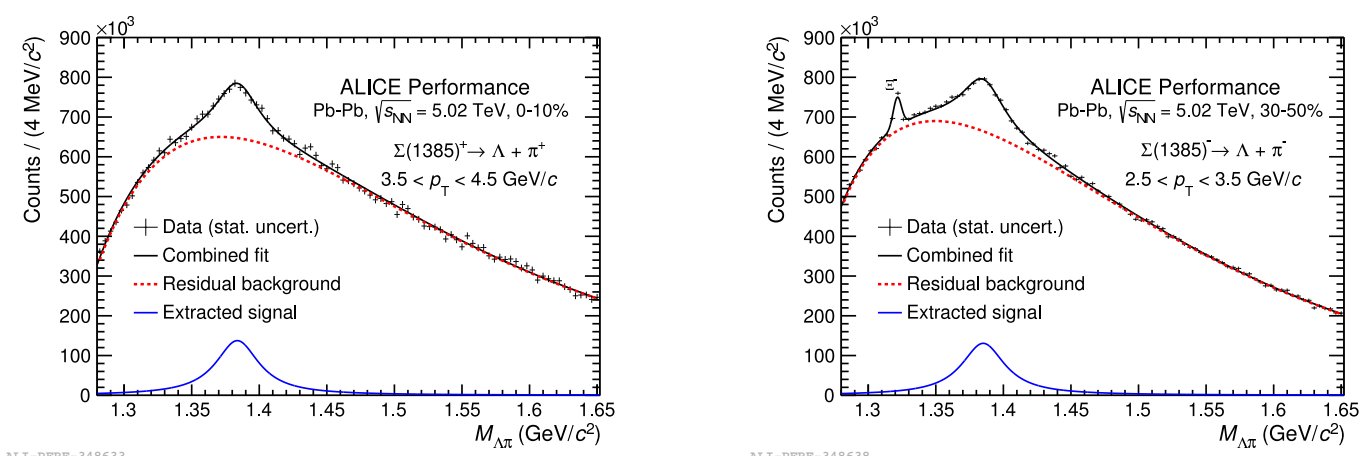

Figure 1: Mixed event subtracted invariant mass distribution of $\Lambda \pi$ pairs (left $\Sigma(1385)^{+}$, right $\left.\Sigma(1385)^{-}\right)$.
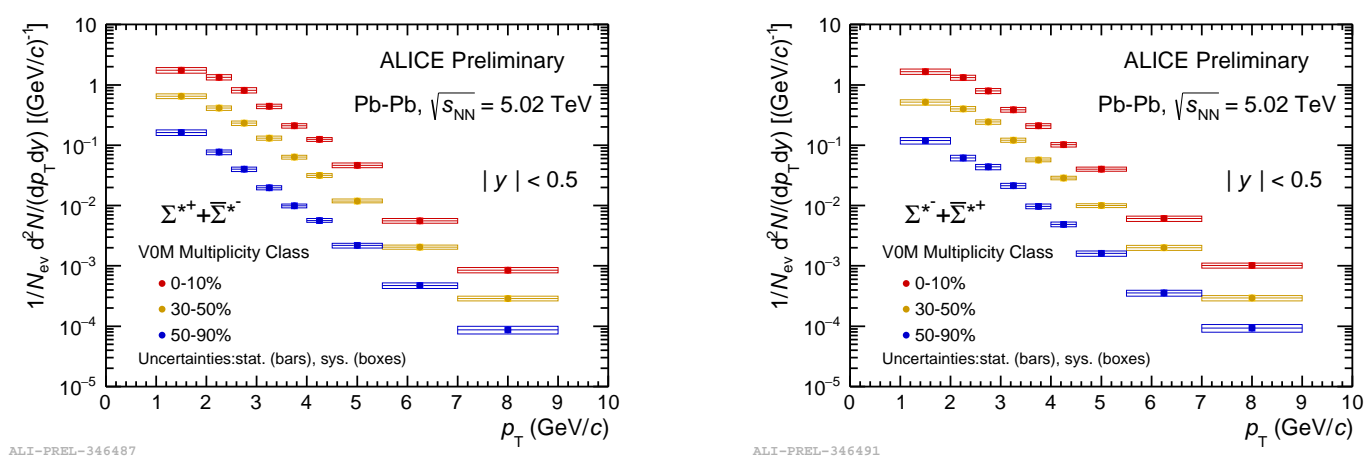

Figure 2: $p_{\mathrm{T}}$ spectra of $\Sigma(1385)^{+}$(left) and $\Sigma(1385)^{-}$(right) resonances in $\mathrm{Pb}-\mathrm{Pb}$ collisions at $\sqrt{s_{\mathrm{NN}}}=5.02 \mathrm{TeV}$ in three centrality classes.

\section{3. $p_{\mathrm{T}}$ spectra in heavy-ion, $\mathbf{p}-\mathbf{P b}$ and $\mathbf{p p}$ collisions}

Most of the resonances mentioned in Table 1 have been measured in heavy-ion, $\mathrm{p}-\mathrm{Pb}$ and pp collisions in order to build a comprehensive picture of their production mechanisms and their evolution in the hadronic medium. In Fig. 2, the recently measured $p_{\mathrm{T}}$ spectra of $\Sigma(1385)^{ \pm}$in $\mathrm{Pb}-\mathrm{Pb}$ collisions at $\sqrt{s_{\mathrm{NN}}}=5.02 \mathrm{TeV}$ are shown in the range $p_{\mathrm{T}}=1-9 \mathrm{GeV} / c$ and in three centrality classes. For all measured resonances, the $p_{\mathrm{T}}$ spectra in $\mathrm{Pb}-\mathrm{Pb}$ collisions show an increasing slope with increasing centrality, an effect of the radial expansion of the system with a common flow velocity. In small systems ( $\mathrm{p}-\mathrm{Pb}$ and $\mathrm{pp}$ ), a similar effect is present which is qualitatively close to what is observed in heavy-ion collisions. This confirms the results obtained from other light-flavour hadrons [11] with the colour reconnection mechanism proposed as a possible explanation of the collective flow-like effects occurring in small collision systems.

\section{4. $p_{\mathrm{T}}$-integrated yields and $\left\langle p_{\mathrm{T}}\right\rangle$}

The evolution of the $p_{\mathrm{T}}$-integrated yields and the $\left\langle p_{\mathrm{T}}\right\rangle$ of the spectra as a function of the charged particle multiplicity provide valuable information about resonance production. Results from measurements of $\mathrm{K}^{*}$ and $\phi$ in pp collisions at $\sqrt{s}=13 \mathrm{TeV}$ and in $\mathrm{p}-\mathrm{Pb}$ collisions at $\sqrt{s}=5.02 \mathrm{TeV}[4,9]$ showed that the production rates of these resonances do not depend on the 
collision system or energy but are driven by the charged particle multiplicity, following a linear increase. This is in agreement with similar measurements from strange hadrons [12]. In Fig. 3, the $p_{\mathrm{T}}$-integrated yields of $\mathrm{K}^{*}$ as a function of the charged particle multiplicity are presented in heavy-ion collisions, showing that the multiplicity drives the produced yield.

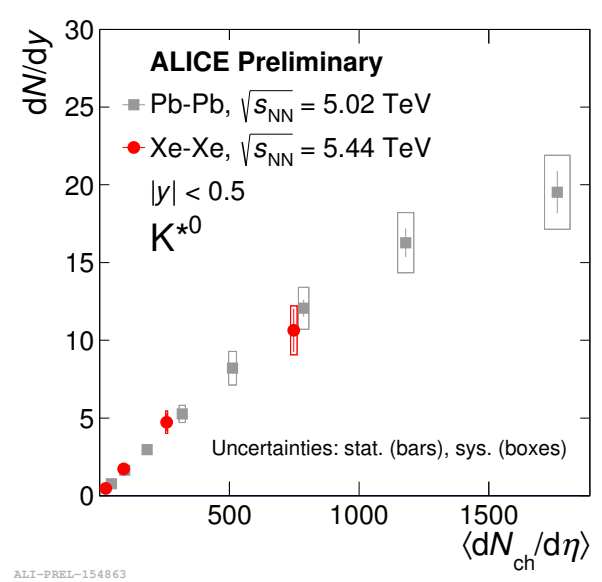

Figure 3: $p_{\mathrm{T}}$-integrated yields of $\mathrm{K}^{*}$ resonance in $\mathrm{Xe}-\mathrm{Xe}$ and $\mathrm{Pb}-\mathrm{Pb}$ collisions at mid-rapidity, as a function of the charged particle multiplicity.

For the $\left\langle p_{\mathrm{T}}\right\rangle$, results from $\mathrm{K}^{*}$ and $\phi$ have demonstrated a faster increase with multiplicity in $\mathrm{pp}$ than in $\mathrm{p}-\mathrm{Pb}$ and $\mathrm{Pb}-\mathrm{Pb}$ collisions. $\left\langle p_{\mathrm{T}}\right\rangle$ in pp follows the same trend regardless the collision energy. The same behaviour is observed in heavy-ions where the $\left\langle p_{\mathrm{T}}\right\rangle$ in $\mathrm{Xe}-\mathrm{Xe}$ and $\mathrm{Pb}-\mathrm{Pb}$ collisions as a function of the charged particle multiplicity follows a common trend (Fig. 4). Comparing $\mathrm{K}^{*}, \phi$ and $\mathrm{p}$, which have similar masses, one observes that all three particles have similar $\left\langle p_{\mathrm{T}}\right\rangle$ in central $\mathrm{Pb}-\mathrm{Pb}$ collisions, but the mass ordering is broken in $\mathrm{pp}, \mathrm{p}-\mathrm{Pb}$ and $\mathrm{Pb}-\mathrm{Pb}$ peripheral collisions. In $\mathrm{Pb}-\mathrm{Pb}$ collisions, the increase in $\left\langle p_{\mathrm{T}}\right\rangle$ is attributed to the radial flow from the strongly-interacting medium, however, the same effect existing in small systems is not yet understood. For pp collisions, comparisons with models show that EPOS-LHC best describes the experimental data, while PYTHIA8 with colour reconnection results in lower $\left\langle p_{\mathrm{T}}\right\rangle$. PYTHIA6 describes the $\phi$, but under-predicts the $\mathrm{K}^{*}$ values [4].

\section{Particle ratios}

Resonances are useful probes to study the hadronic phase and to define the strength of rescattering and regeneration effects. Figure 5 shows the ratio of $\Sigma(1385)^{ \pm}$resonances to the stable $\Lambda$ baryon in all available collisions systems in ALICE and STAR experiments. Unlike the EPOS event generator with UrQMD afterburner and thermal model predictions, it is observed that $\Sigma(1385)^{ \pm}$ yields are suppressed going to the most central collisions.

In Fig. 6, the ratio of resonances over longer-lived hadrons with the same strangeness are presented for resonances having different lifetimes. Except for the $\Xi(1530)^{0}$ and $\phi$ which have larger decay times, short-lived resonances $(c \tau<13 \mathrm{fm} / c)$ exhibit a suppression in heavy-ion collisions comparing to the pp ones. $\rho$ and $\mathrm{K}^{*}$ seem to be suppressed in high multiplicity pp 

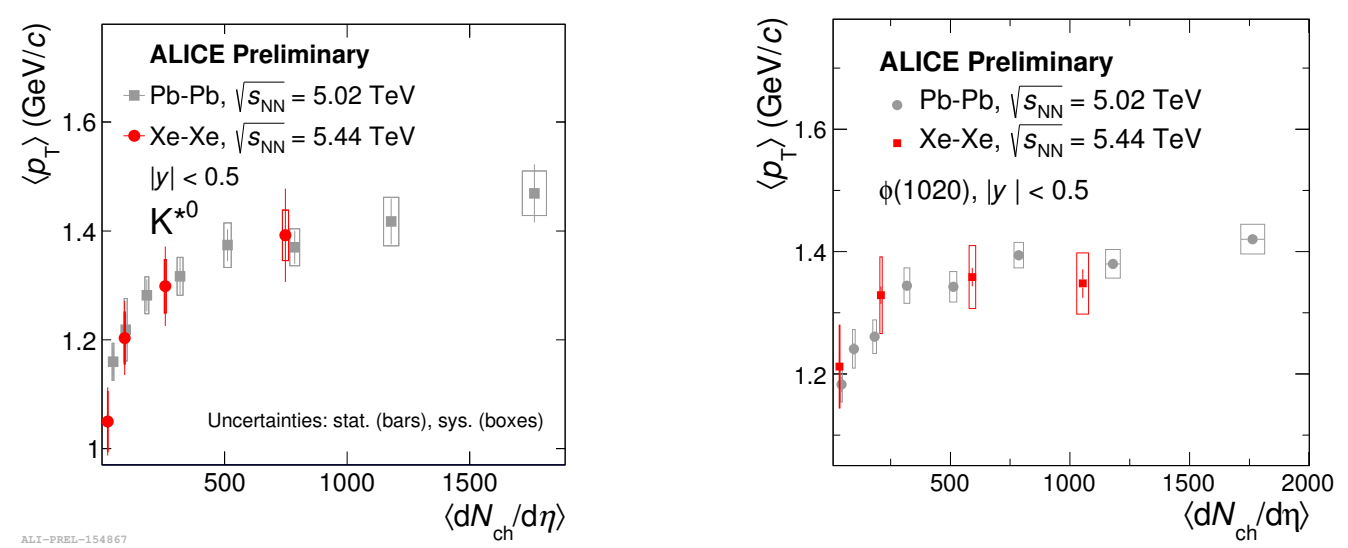

Figure 4: $\left\langle p_{\mathrm{T}}\right\rangle$ of $\mathrm{K}^{*}$ and $\phi$ as a function of the charged particle multiplicity in heavy-ion collisions.

collisions but the systematic uncertainties do not allow a definite conclusion. In most cases, EPOS describes qualitatively the measured trend but fails to predict the ratios quantitatively [3].

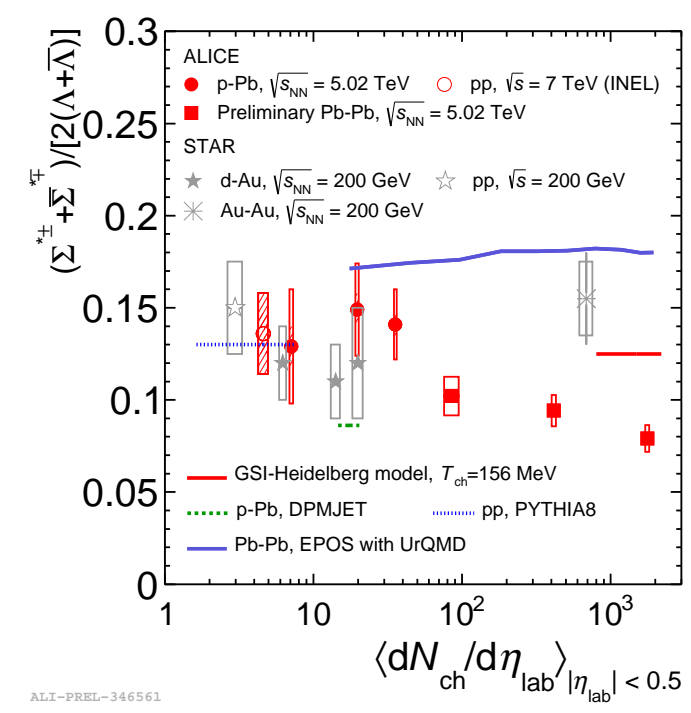

Figure 5: Ratio of the number of $\Sigma(1385)^{ \pm}$resonances to the number of long-lived $\Lambda$ baryons in $\mathrm{pp}, \mathrm{p}-\mathrm{Pb}$ and $\mathrm{Pb}-\mathrm{Pb}$ collisions in ALICE, compared to results from the STAR experiment and theoretical models.

\section{Summary}

Results from hadronic resonances reconstructed and identified by the ALICE experiment have been presented. In central $\mathrm{Pb}-\mathrm{Pb}$ collisions, the production of resonances follows hydrodynamic behaviour observed for the other light particles. The study of the multiplicity dependence of resonance production in small systems shows a similar bahaviour to the $p_{\mathrm{T}}$ evolution seen in heavy-ion collisions. The new measurement of $\Sigma(1385)^{ \pm}$resonances has been shown, with the $\Sigma(1385)^{ \pm} / \Lambda$ ratio exhibiting a suppression going from peripheral to the most central collisions. 


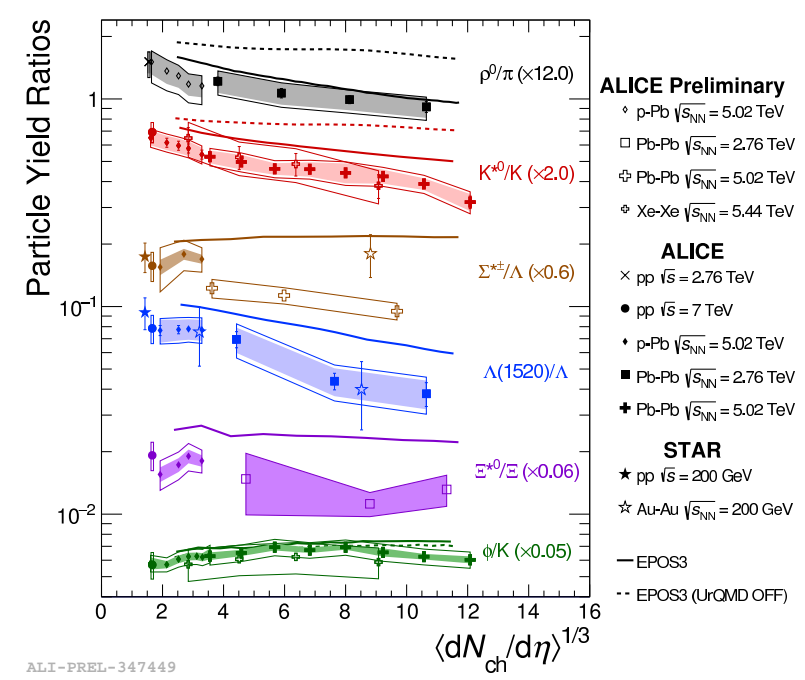

Figure 6: Resonance to the non-resonant particle yield ratios in $\mathrm{pp}, \mathrm{p}-\mathrm{Pb}, \mathrm{Pb}-\mathrm{Pb}$ and $\mathrm{Xe}-\mathrm{Xe}$ collisions in ALICE, for all measured resonances by the experiment together with the EPOS with and without UrQMD predictions.

This preliminary result is consistent with the suppression of $\mathrm{K}^{*}$, which has a similar lifetime to $\Sigma(1385)^{ \pm}$and suggests stronger re-scattering than regeneration in the hadronic phase. ALICE is currently exploring other resonances like $f_{0}(980)$ and $\Xi(1820)$, for which very little information exists regarding their production and/or properties.

\section{References}

[1] N. Cabibbo and G. Parisi, Phys. Lett. 59B (1975) 67-69.

[2] E. Laermann and O. Philipsen, Ann. Rev. Nucl.Part. Sci. 53 (2003) 163-198

[3] ALICE Collaboration, S. Acharya et al., Phys. Lett. B802, 135225 (2020)

[4] ALICE Collaboration, S. Acharya et al., Phys. Lett. B807, 135501 (2020)

[5] ALICE Collaboration, S. Acharya et al., Phys. Rev. C 102, no.2, 024912 (2020)

[6] ALICE Collaboration, S. Acharya et al., Eur. Phys. J. C 80, no.2, 160 (2020)

[7] ALICE Collaboration, S. Acharya et al., Phys. Rev. C 99, no.6, 064901 (2019)

[8] ALICE Collaboration, J. Adam et al., Phys. Rev. C 95, no.6, 064606 (2017)

[9] ALICE Collaboration, J. Adam et al., Eur. Phys. J. C 76, no.5, 245 (2016)

[10] ALICE Collaboration, B. Abelev et al., Int. J. Mod. Phys. A29, 1430044 (2014)

[11] ALICE Collaboration, S. Acharya et al., arXiv:2005.11120 [nucl-ex].

[12] ALICE Collaboration, S. Acharya et al., Eur. Phys. J. C 80, no.2, 167 (2020) 